La mécanque rationnelle ( ${ }^{\text {re }}$ et $2^{\mathrm{e}}$ année) se complète d'un cours de mécanique appliquée où sont étudiés au point de vue pratique les engrenages, les transmissions et les transformations de mouvements.

L'arpentage et le nivellement ( ${ }^{\mathrm{re}}$ année) font le sujet d'un certain nombre de leçons spéciales, et les élèves appliquent sur un terrain varié, sous la direction de leur professeur, et de conducteurs de Ponts et Chaussées, les notions qui leur ont été données; le nombre des séances d'application est suffisant pour que chaque élève se serve de tous les instruments employés au lever de plan.

La physique générale est enseignée en première année, sauf la partie relative à l'électricité générale ( $2^{\circ}$ année). Tout en mettant les élèves au courant des théories nouvelles sur les différentes formes qu'affecte l'énergle et les applications qui en découlent, le cours sert d'introduction à la physique industrielle et à la métallurgie ( $2^{\mathrm{e}}$ année). L'ensergnement de ces deux dernières parties est complété par des visites dans les usines métallurgiques de la région.

La chimie minérale ( $I^{\text {re }}$ année) et la chimie organique $\left(2^{e}\right.$ année) donnent aux futurs ingénieurs les notions générales qui leur sont nécessaires dans leur carrère. De même, l'étude de la minéralogie ( $1^{\mathrm{e}}$ année) et de la géologie ( $2^{\mathrm{e}}$ année) les mettent à même d'étudier la nature du terrain pour le tracé d'une route, d'un chemin de fer ou d'une canalisation hydraulique.

La troisième année est réservée aux applications générales dont tout ingénieur a besoin, quelle que soit la voie qu'sl adopte dans la suite. Les cours de construction de machines, d'hydraulique, de résistance des matériaux, de statique graphique, de machine à vapeur, d'électrotechnique générale, d'analyse chimique, de législation industrielle, de patholocie d'urgence, forment un ensemble de connaissances qui facilite l'orientation défnitive, des élèves et qui leur permet de se créer par leur travail une situation dans l'industrie.

Ici encore, on s'efforce, chaque fois que la chose est poss1ble, de faire intervenir l'expérience : les élèves vérifient les notions données aux cours en se servant de la machine à essayer les métaux, en prenant des diagrammes, et en faisant des essais au frein sur la machine à vapeur, en effectuant au laboratoire d'électrotechnique des mesures de résistance, de force électromotrice, de capacité, étalonnage de compteurs et d'appareils industriels.

A la fin de la deuxième année et pendant toute la troisième année, les élèves sont tenus de faire les projets suivants : physique industrielle, machine à vapeur, statique graphique, résistance des matériaux, hydraulique, constructions civiles, travaux publics.

Des visites d'usines viennent à la fin de leur séjour à l'Ecole pendant le dernier trimestre, compléter l'ensemble de leurs études, leur montrer la diffénence existant entre l'Ecole et l'usine et leur donner un aperçu de la nouvelle voie dans laquelle lls auront à s'engager.

Année de spécialisation. - La quatrième année, ou année de spécialisation, est de création récente. C'est en novembre 1902 que, grâce à une subvention donnée spécialemient dans ce but par le Conseil général du Rhône, on a organisé tout un ensemble de cours sur l'électrotechnique et la mécanique appliquée (section $\mathrm{A}$ ), et en novembre 1905 s'ouvraient de nouveaux cours permettant aux élèves d'étudier plus spécialenent les constructions civiles et les travaux publics (section $\mathrm{B}$ ).

Dans ces années de spécialisation, on s'efforce de rendre l'enseignement aussi pratique que possible : la matinée est consacrée aux leçons orales, et la soirée aux travaux pratiques de laboratorre et d'ateliers, aux projets de moteurs, de dynamos, d'installations électriques, de transports d'énergie, de turbines, aux visites d'usines électriques, de chantiers, etc. Des stages sont faits à la fin de l'année dans différentes usines et installations électriques, exploitations et chantiers, de manlère que les connaissances acquises tant aux cours que dans les laboratoires reçoivent la consécration industrielle.
Les leçons sur l'aménagement des chutes d'eau, le transport de l'énergie électrique, l'étude des turbines à vapleur, la sur. chauffe, l'aménagement des centrales électriques, la traction élctrique, l'application de l'électricité aux machines-outıls et apparels de levage sont communes aux deux sections.

A la section A des cours spéciaux sont faits sur des con: pléments de mathématiques ct de mécanique rationnelle, sur la thermodynamıque, l'électrotechnique générale, les mesures électriques, les essais de machines, le calcul et la construction des machines à courant continu, le calcul des transformateurs, alternateurs ou alternomoteurs, la construction de câbles armés, les canalisations souterraines, les essais des machines à vapeur, des chaudières et des combustıbles, l'électrochimie et l'électrométallurgile, la photométrie.

Pour la section $\mathrm{B}$, on a créé des cours de géologie, topographie, géodésie, construction de routes, ponts en maçonnerie, ponts en bois, ponts en fer, fonte, béton et ciment armé, maison d'habitation, construction de chemins de fer, exploitation technique. Ce sont les cours de cette section que suivent les ingénieurs coloniaux.

En résumé, les sections $A$ et $B$ ont sept cours communs t chacune a onze cours spéciaux.

Dans ces années de spécialisation, dans lesquelles on serre de très près la réalité, les élèves peuvent donc acquérir un ensemble de connaissances essentiellement pratıques et immédiatement applicables. L'enseignement technique colonıl permettra à de jeunes ingénieurs de se créer rapidement d'avantageuses situations dans les pays encore neufs au point de vue de la grande industrie.

Tel se présente, avec ses 33 professeurs, ses 6 chefs ouvriers, ses I 70 élèves, l'enseignement donné à l'Ecole Centrale Lyonnaise. Si, peu à pieu, l'Ecole est arrivée à un tel développement, et s'il lui est permis d'adapter sans cesse ses cours aux nouveaux desiderata de l'industrie en marche, c'est que, jamais, à aucun moment, la bonne volonté de tous ne lui a fait défaut : industriels, commerçants, pouvoirs publics, tous ont contribué, chacun dans sa sphère, à soutenir l'Ecole et lui ont permis d'évoluer dans le sens du progrès.

L'Ecole, très reconnaissante, fait en sorte de remplir les vues des fondateurs et donateurs en apportant sa part d'efforts dans la lutte industrielle.

\section{H. Rigollot,}

Professeur adjoint à la Faculté des Sciences de I'Umversite de Lyou Duectour de l'Ecole Centrale Lyountise

\section{Premier Congrès National} $\mathrm{DE}$

\section{NAVIGATION INTERIEURE}

La Société du Sud-Ouest Navigable, profitant de la tenue à Bordeaux, en 1907, de la première Exposition Maritiro Internationale, avait décidé d'organiser, à cette occasion, lo Premier Congrès National de Navigation Intérieure, sous le patronage de la Chambre de Commerce de Bordeaux et des Autorités constituées de cette ville et du département de la Gironde.

Ce Congrès devait être national, c'est-à-dire qu'il devait traiter des sujets intéressant la France entière. Il devait avoir pour but d'étudier et de discuter les questions générales et, plus spécialement, celles qui lui paraîtraient d'un intérèt primordial pour la Navigation intérieure en Erance, au début du XXe siècle.

Nous nous proposons, dans cet article, de donner un court résumé des rapports qui ont été présentês à ce Con. grès, ainsi que des discussions et des vœux qui en ont été la suite et la conclusion.

Le premier Congrès National de Navigation Intérieure s'est ouvert à Bordeaux le jeudi 18 juillet, à 9 heures du 
matin, dans la grande salle des fêtes de l'Exposition, sous la présidence de M. BEsse, président de la Chambre de Commerce de Bordeaux.

Pour montrer l'intérêt qu'il portait aux travaux de ce Congrès, le Gouvernement s'y était fait représenter par MM. Mengrn-Lecreulx, inspecteur général des Ponts et Chaussées; VIDAL, ingénieur en chef des services maritimes de Bordeaux; DABAT, directeur de l'hydraulique agricole au ministère de l'Agriculture, et MERCADET, chef de bureau au ministère du Commerce et de l'Industrie.

Dans son discours d'inauguration, $M$. Besse examine la valeur de l'argument qui oppose les intérêts des chemins de fer à ceux de la navigation intérieure. Les adversaires de celle-ci considèrent les voies fluviales comme inutiles dans l'économie moderne des transports. D'autres craignent que la concurrence ne soit pour les chemins de fer une cause de diminution de recettes, et qu'elle ne nuise ainsi aux intérôts généraux du pays, et à ceux du Trésor public. Les uns et les autres ne peuvent pas avoir oublié que trop souvent, depuis quelques annees, les chemins de for ont été impuissants à faire face aux besoins toujours croissants de lagricullure, de l'industrie et du commerce. Ils ne peuvent pas ignorer que les prix des transports sont très élevés sur celles de nos voies ferrées qui ne redoutent pas la concurrence des rivières et des canaux.

Alors ne doivent-ils pas être conduits à conclure qu'une batellerie bien organisée, sur des voies d'eau rationnellement aménagées, ferait pénétrer à bon compte dans l'intérieur du pays des marchandises de gros tonnage, et de faible valeur, qui ne peuvent supporter les frais de la voie ferrée, et qu'elle fournirait ainsi à l'agriculture et à l'industrie les moyens d'accroitre, en développant leur production, l'élément transportable capable de payer un prix plus élevé?

Comme preuve que les chemins de fer, loin d'avoir á redouter la batellerie, trouvent profit à son progrès, loraleur cite l'exemple de l'Allemagne, oú les recettes des Compagnies ont constamment grandi à mesure que l'amélioration du réseau de navigation intérieure facilitait un plus grand développement de l'agriculture et de l'industrie.

D'un autre côté, pour voir combien la coopération de la navigation fluviale et de la navigation maritime peut être féconde, ne suffit-il pas, ajoute M. Besse, de jeter les yeux sur limmense et si rapide essor que les transformations du Rhin, de l'Elbe et de leurs affluents, on tonné aux ports de Rotterdam et de Hambourg? En présence de ces merveilleux résultats, ne peut-on pas affirmer, avec M. Louis Lalfitte, que lo trafic d'un port est en raison direcle de l'importance de la voie navigable sur laquelle il est sitú́, et de la région dont cette voie draine les produits.

Sans doute, il n'y a pas apparence que, de longtemps, le budget de la France lui permette de donner à son réseau de navigation intérieure le milliard que l'Allemagne a donné au sien; mais, peut-être, sous l'impulsion des Chambres de commerce, des municipalités, pourrait-on trouver des capitaux, collectifs ou privés, qui suppléeraient à l'impuissance de l'Etat. Malheureusement, nous nous heurtons à cette centralisation déprimante, à cette tutelle oppressive, qui étouffe toute initiative dans son germe, ou qui l'empêche d'aboutir, si elle a eu assez de force pour se montrer.

M. Besse termine ainsi son discours : "Donc, Messieurs, poursuivre l'amélioration des voies d'eau naturelles et artificielles actuellement existantes; étudier la réalisation possible de nouveaux ouvrages qui mettent en communication les divers bassins français, tel est, résumé en deux phrases, le programme de vos travaux $)$.

Après avoir déclaré ouvert le premier Congrès National de Navigation Intérieure, M. BESSE invite les congressistes à nommer leur bureau, qui est ainsi constitué : Président : M. E. Buhan, président du comité de Bordeaux du Sud-
Ouest Navigable; vice-présidents : MM. CoLson, conseiller d'Etat; Linyer, avocat, président du comité de la Loire Navigable, QuinetTe de Rochemond et PARLIER, inspecteurs généraux des Ponts et Chaussées; FeugA, président du comité de Toulouse du Sud-Ouest Navigable; Lefebvre, vice-président de la Chambre de Commerce de Paris; DE SAINTIGNon, membre de la Chambre de Commerce de Nancy; secrétaire général : M. A. RöDEL, industriel à Bordeaux, secrétaire général du comité d'organisation du Congrès. Parmi les secrétaires, signalons notre collaborateur, M. P. Buffault, inspecteur des Eaux et Forêts à Périgueux.

En prenant possession du fauteuil présidentiel, M. BuHAN déclare tout d'abord que ce Congrès a un but bien distinct de celui des Congrès rêgionaux et des Congrès internationaux. Il précisera quelle part doit revenir à la voie navigable dans les transports de notre pays. La variété des solutions à donner aux questions de navigation intérieure, suivant les pays, offre aux Congrès nationaux un champ considérable à exploiter. Seul. le Congrès national nous renseignera sur le développement à donner au réseau, en tenant compte des besoins et des facultés financières du pays. Seul, il sera à même d'amener le grand public à s'intéresser aux choses de la navigation, et à exercer sur les pouvoirs publics une action effective.

L'orateur reconnait, d'ailleurs, que cette cuvre ne peut être celle d'une première session, et qu'elle ne peut être menée à bien que par une succession de réunions périodiques. Il ajoute qu'il appartient au Congrès actuel de donner à cette tentative l'essor qui doit la r'endre féconde en s'appliquant à donner à ses travaux un caractère vraiment national, en s'êloignant à la fois des discussions qui ne seraient pas étroitemenl liées au sort général du réseau français, et de celles qui, s'égarant à traiter des besoins locaux, viendraient usurper la place des Congrès régionaux, et enlever à cette manifestation sa véritable portée.

M. Buhan ajoute que, très souffrant, il serait reconnaissant au Congrès de bien vouloir le décharger du soin de diriger ses travaux. Sur sa demande, le Congrès invite M. Linyer à remplir les fonctions de président.

Les travaux du Congrès débutent par la lecture du rapport de M. LAFFITTE, secrétaire général de la Chambre de Commerce de Nancy, commissaire enquêteur de la Loire Navigable, sur la Condition du réseau navigable francais (Sa comparaison au point de vue technique et économique avec les principaux Etats européens. Rôle économique do la batellerie moderne).

On a fini par reconnaitre que, dans l'Etat moderne, le réseau navigable forme une des pieces principales du mécanisme économique. Le développement de la navigation intérieure, comme celui des chemins de fer, doit permettre aux Etats producteurs, soit daméliorer la condition do l'industrie, soit de multiplier les achats et les ventes. De nos jours, la batellerie doit participer assez activement a la vie nationale pour favoriser l'abaissement des prix de revient et l'extension du marché extérieur. Mais il faut aussi qu'elle évolue, qu'elle marche avec le progrès, en perfectionnant son matériel et ses méthodes, afin que ses aptitudes soient en harmonie avec celles de ses collaborateurs ou de ses concurrents.

Les voies artificielles accomplissent donc, chez nous, une fonction de premier ordre. Le trafic auquel elles se prêtent représente un élément considérable de la circulation intérieure Etant donné leur rôle, elles devraient être tou jours en état de satisfaire aux exigences du progrès économique. L'expérience démontre qu'il n'en est pas ainsi. Maintenue dans une condition inférieure par les limites fixes des écluses, par une exploitation dépourvue de souplesse, et par une organisation commerciale insulfisante, la batellerie du Nord n'a pu, ces derniers temps, résister à la concurrence du chemin de fer. 
Il faudrait que la capacité de nos canaux, et celle du matériel qui les utilise, augmentât en même temps que le trafic se développe. Cette condition est réalisable sur les fleuves. Parmi les causes du merveilleux essor de la batellerie allemande, il faut compter l'aménagement progressif des longs cours deau qui constituent la partie principale du réseau navigable de l'Empire, et surtout la facilité avec laquelle nos voisins ont pu, à mesure que le commerce le demandait, accroitro les dimensions des véhicules.

Depuis longtemps, on a sigualé l'inégale répartition des voies, et l'inégale distribution du trafic qui en est la conséquence. Il y a plus de soixante ans, Michel Chevalier notait le contraste que présentaient, d'un côté, le Nord et l'Est, richement pourvus par l'Etat de routes de toute sorte; de l'autre, l'Ouest, abandonné à lui-même. "Entre les deux moitiés de France, écrivait-i1, le partage des travaux publics semble avoir été fait d'après le principe de ce seigneur féodal qui avait pris pour devise : « Tout d'un côté et rien de l'autre ". S'il revenait, cet économiste pourrait renouveler sa boutade. De nombreuses raisons, économiques ou autres, ont, jusqu'à notre époque, détourné de celte région déshéritée l'attention des pouvoirs publics et les disponibilités budgétaires. L'iniliative privée, il est vrai, s'est efforcée, dans la dernière décade, de réagir et de secouer l'indifférence des intéressés. L'œuvre entreprise avec succès dans les départements de l'Ouest et du centre par la Société la Loire navigable a été étendue par le SudOuest Navigable aux pays de la Garonne.

Les régions comprises entre la Loire, le Rhône et l'Atlantique étant encore dépourvues d'un réseau homogène et bien organisé, pourront être le champ d'expérience des techniciens et des entrepreneurs de transport. Ces régions, parce qu'elles auront longtemps attendu l'outillage dont elles auront besoin, bénéficieront des plus récents progrès scientifiques.

Il apparait bien que la batellerie du Sud-Ouest ne joue qu'un ròle insuffisant. Dotée d'un réseau équivalant aux 23,28 centièmes du réseau français, elle ne transporte que 2 millions 49635 tonnes, soit $6,02 \%$ du tonnage véhiculé par eau, dans la France entière. Pourquoi, dès lors, s'éton ner de la stagnation du commerce maritime de Bordeaux? Si les voies qui relient ce port à sa zône de drainage n'accomplissent qu'en parlie leur fonction, le port lui-même, privé d'aliment, languit et dépérit. C'est, en effet, dans nos places maritimes que se remarque le plus aisément l'insuffisance générale de notre réseau navigable. Puisque le commerce maritime est, de nos jours plus que Jamais, le résumé de la vie nationale, la batellerie, organe du corps économique, doit participer à la vie des ports dans la même mesure où ceux-ci collaborent a la circulation générale. Son rôle dans l'approvisionnement de ces places, ou dans l'ćvaluation des marchandises sera, en quelque sorte, la vérification de sa fonction. Le rôle de la batellerie, considérée comme auxiliaire du commerce de mer, est très actif, le fait est bien connu, dans les ports étrangers con currents des nòtres : $\dot{a}$ Hambourg, à Rotterdam, à Anvers. Il est à peu près nul à Marseille, notre premier port maritime. Il n'a quelque importance qu'à Dunkerque, et à Rouen,que desservent les meilleures voies de notre réseau, et qui, de ce fait, ont progressé plus vite que les autres ports français, dans le quart de siècle écoulé.

Hambourg est prospère grảce à l'Elbe, Rotterdam grâce au Rhin, Anvers grâce au Rhin et à l'Escaut. Il dépend donc de nous que nos places maritimes deviennent les heureuses concurrentes d'Anvers, de Rotterdam, et de Hambourg, avec l'aide d'un réscau navigable définitivement rendu à ses destinées.

Dans l'après-midi du mème jour, le Congrès s'est réuni, ainsi qu'il l'a fail d'ailleurs pour ses séances ultérieures, dans le pavillon de la Bolgique, monument de style gothique construit sur le bord même de la Garonne, et représentant le Steen d'Anvers.

M. R. TAVERnier a donné lecture d'un rapport de M. Barattier De Mas, inspecteur général en retraite, ef ancien professeur du cours de navigation à l'Ecole Natio. nale des Ponts et Chaussées, sur l'Aménagement et lExploitation du réseau navigable francais.

A la suite de ce rapport, le Congrès a émis le vœu sui. vant, présenté par M. DE MAS comme conclusion à son étude, et amendé par M. R. TAvernier et par M. PÉRIER DE FÉrAl, président de la Chambre Syndicale de la Marine: " $1^{\circ} Q u^{\prime}$ 'en ce qui concerne les rivières à courant libre, l'effet des travaux de régularisation soit complété par des dragages périodiques et que, dans ce but, les serviceś de navigation intéressés soient pourvus d'un matériel de dragage approprié.

“ 20 Qu'en ce qui concerne les rivières canalisées, sur les lignes les plus importantes et de transit, la dimension minima des écluses soit fixée à 190 mètres de longueur sur 11 mètres de largeur, pour permettre d'y faire passer un convoi entier avec son remorqueur et par suite. en pratique des embarcations de longueur et de largeur quelconque.

« $3^{\circ}$ Qu'en ce qui concerne les canaux :

( $a)$. Au point de vue de la construction, on adopte dans l'avenir, pour les parties comprises dans certaines lignes de très grand trafic et de transit, des dimensions qui permettent le passage de bateaux susceptibles de porter 500 à 600 tonnes de marchandises, avec des formes favo. rables à une traction économique, c'est-à-dire mesurant al minimum 60 mètres de longueur sur 8 mètres de largeur.

«b). Au point de vue de l'exploitation, on se préoccupe d'assurer aux entreprises de traction mécanique les condi. tions d'existence qui en favorisent le développement. ")

D'autre part, sur la proposition de MM. BOILÈVE, DEBOH. Chaud, de Peyraud, Doumerc, Delboy, Schwob, Laval, etde notre collaborateur le commandant AUdEBRAND, le Congres signale à l'attention des Pouvoirs Publics l'utilité qu'il y aurait à donner lo plus tòt possible une sanction définitive.

“ $1^{\circ}$ Aux études relatives au canal latéral au Rhône pout l'irrigation et la navigation; (*)

" $2^{\circ}$ Aux études poursuivies depuis de longues années pour la meilleure utilisation des eaux de la Loire;

" $3^{\circ}$ Aux projets qui ont été mis en avant pour joindpe le réseau navigable du Sud-Ouest avec celui du Centre, de l'Est et du Sud-Est, et faire un choix judicieux dans le plus bref délai ».

Le même jour, notre éminent collaborateur, M. FABRe: inspecteur des Eaux et Forêts, a donné lecture d'un important travail sur la Restauration des montagnes et la navigation intérieure en France.

Les faits discutés au cours de ce rapport sont limitési à ceux intéressant les bassins de nos trois grands fleuves montagneux : le Rhône, la Garonne et la Loire; leur enchal nement est le suivant:

I. La montagne, génératrice d'énergie, alimente nos rivières et en régularise le régime par l'armature végétale de ses versants.

II. Une lutle incessante et progressive se livre d'amonl en aval, dans chaque hassin, entre le troupeau qui dénude et mobilise le sol montagneux, et la drague qui cherched parer aux méfaits de cette dénudation.

III. La marche du sol à l'aridité est constante par le fail de la dénudation.

IV. Le remède ne saurait être dans l'emmagasinement des crues torrentielles, mais plutòt dans l'aménagement des eaux superficielles, surtout par la végétation.

(1) On aurait pu ajouter la force molrice, de manıère à utiliser la dénivellation des écluses pour produire de lénergre électrique qui sent utilisée pour la traction mécanique des bateaux. 
V. La restauration, et du sol montagneux et du régime de nos rivières, est subordonnée à la protection rigoureuse du sol, à la source des eaux. C'est vers rette protection que doit évoluer notre législation sylvo-pastorale.

Comme conclusions à cctte étude, M. Fabre propose au Congrès d'émettre le vou suivant :

( $1^{\circ}$ De signaler à la Société Nationale d'agriculture, à la Société des Agriculteurs de France, aux Conseils généraux, aux Chambres de Commerce, aux Académies régionales et aux Sociétés savanles, aux Conseils d'hygiène, aux Syndicats d'initiative..., les dangers progressifs que la dénudation des montagnes, la déforestation en général, la dévastion des clâtaigneraies, font courir au régime des rivières et des eaux superficielles, à l'irrigation, à l'hydraulique, à lia sécurité, à l'alimentation, et à l'hygiène publiques, à l'es thétique du pays;

"De solliciler des voux motivés adressés aux pouvoirs publics et au Parlement, en vue d'assurer la protection du Sol;

“20 De poursuivre le groupement, au Parlement, d'initiatives indépendantes pour élaborer une législation protectrice du sol, en faire naitre et en suivre la discussion, assurer dans la suite le contrôle parlementaire que comportera l'exercice de cette loi, et ses modifications s'il y a licu».

Le commandant Audebrand combat cette dernière conclusiois, car il redoute l'intervention de l'Elat. Timeo Danaos et dona ferentes. C'est à l'Initiative privée qu'il faut avour recours, si l'on veut restaurer les montagnes. D'ailleurs, il existe ces lois suffisantes, il n'y a qu'à les appliquer.

M. Fabre répond au commandant Audebrand qu'il est aussi individualiste que lui, et aussi partisan de l'initiative privée, mais, étant donné la pitoyable situation de nos montagnes, il ne voit pas le moyen d'y porter remède sans le concours de l'Etat. M. Fabre déclare qu'il est « étaliste malgré lui

Le commandant Audebrand réplique en citant les excellents résultats de début que l'Association dauphinoise pour l'aménagement des montagnes, dont il est le président, a obtenu par l'initiative privée dans les Alpes $\left({ }^{*}\right)$.

M. Descombes, président de l'Association pour l'aménagement des montagnes, rappelle les catastrophes dont cinq villages français ont été les victimes à la suite du déchâ̂nement d'avalanches de neige de l'hiver dernier. Il importe d'avoir ces faits présents à la mémoire, au moment où il est question de défendre la montagne contre les entreprises des berger; et de leurs troupeaux.

Finalement, le Congrès adopte le Voeu de M. Fabre, tel qu'il laa présenté.

Le vendredi matin, 19 juillet, M. R. TAVERNIER, ingénieur en chef des Ponts et Chaussées, ingénieur conseil de l'Office des Transports (organisé par les Chambres de Commerce du Sud-Est), fait part de son rapport sur les Moyens à employer pour intéresser les capitaux, collectifs ou privés, à la mise en valeur des voies navigables.

C'est une étude très approfondie de la question. L'auteur y examine les efforts tentés pour l'organisation commerciale des voies navigables, d'abord en France, puis en Allemagne, enfin en Belgique. Dans un chapitre final, il résume et compare les renseignements recueillis, et dégage les conclusions pratiques suivantes:

$1^{\circ}$ Dépenses de batellerie. Sous le régime de la liberté de la batellerie, étant donné en France l'importance prédominante des petits bateliers, on intéressera les capitaux privés au dévelıppement de la flotte de navigation intérieure en

(*) Volr dans La Hou lle Blanche d'aô̂t 1907, p. 173, l'article du commandant Audebrand sur la houille blanche et la question sylvopastorale. organisant des services collectifs de tractiou, et des ports publics bien outillés pour les manutentions, les transbordements, les dépôts et les magasinages;

$2^{\circ}$ Dépenses de ports. Les ports publics peuvent être créés, soit à laide des capitaux privés, de concessionnaires ou permissionnaires, soit à l'aide de capitaux directement fournis par les collectivités intéressées : Etat, Départements, Villes, Chambres de commerce;

3o Administration des ports. Dans le second cas, comme dans le premier, des administrations locales, analogues pour le moment à celles des ports de mer, destinées par suite à bénéficier d'un régime d'autonomie plus large, où les droits de gestion seraient proportionnés aux concours fournis, gèreront les entreprises directcment exécutées par leurs soins, prépareront ou surveilleront les concessions, étudieront toutes les mesures propres à faciliter le développement du trafic sous toutes ses formes, tout en assurant aux capitaux employés une productivité convenable, veilleront, en particulier, en réservant les emplacements nécessaires, à ce que les installations privées puissent con. tinuer à se développer à côté des établissements publics :

$4^{\circ}$ Administration des voies navigables. Les dẻlégués des "administrations de ports ", groupés avec les représentants de la batellerie, serviront a constituer, dans chaque région, des " administration:s de voies navigables", qui coordnnneront et complèteront les efforts des a administrations de ports ", pareront aux lacunes et aux insuffisances de l'exploitation, organiseront des services publics de traction partout ou cela sera utile;

$5^{\circ}$ Comilé consultatif des voies na ígables. Ce Comité, recruté en majeure partie parmi les membres des administrations régionales dont il est parlé ci-avant, assistera l'Etat dans l'exercice de ses droits de police et de surveillance. Il étudiera notamment les mesures propres à augmenter le trafic général du réseau, préparera les bases des concours financiers aux travaux neufs d'extension ou d'amélioration, cherchera à créer, entre les diverses administrations régionales, la solidarité financière utile à l'exécution des ceuvres communes.

A la suite du rapport de $\mathrm{M}$. Tavernier, le Congrès : * Considérant que, pour permettre à la batellerie d'améliorer et d'augmenter son matériel. il est indispensale de créer des ports publics bien outillés, et des services collectifs de traction;

"Que, pour provoquer en vue de ces créations les concours utiles, il importe de recouril à des administrations locales autonomes, en proportionnant les pouvoirs de gestion aux concours fournis et ménageant entre elles les solidarités utiles ;

«Que, des projets de loi ont été précédemment proposés, ou étudiés, concernant l'administration locale des ports et des voies navigables, et qu'il convient d'aboutir sans plus de retard aux solutions réalisables en tenant compte des objections soulevées et respectant les situations acquises;

"Qu'un Comité consultatif de Navigation intérieure a été créé dont il importe d'assurer le fonctionnement régulier; )

Emet le vœu: « $1^{\circ}$ Que les ports de Navigation intérieure, sous le contrôle supérieur de l'Etat, soient améliorès, outillés ou exploités par des administrations locales recrutées parmi les collectivités ou les particuliers qui auront contribué aux dépenses;

- 2० Que, partout où ce sera nécessaire, les entreprises indispensables à lexploitation commerciale des voies navigables, notamment les services collectifs de traction, soient organisés par des administrations régionales où seraient représentées les administrations des ports riverains et la batellerie;

" $3^{\circ}$ Que le Comité consultatif de Navigation soit mis en situation de fonctionner régulièrement, et qu'il soit recruté en majeure partie, quand cela deviendra possible, parmi les 
membres des administrations locales ou régionales chargées d'assurer l'exploitation commerciale des ports et des voies navigables, et parmi les membres de la batellerie ".

M. Maurico Schwob, directeur du Phare de la Loire, fait ensuite une cormmunication sur l'Utilisation des Eaux courantes, au point de vue de l'agriculture, de l'industrie et de la navigation. Cette communication ayant été insérée in extenso dans La Houille Blanche de janvier 1908, nous y renverrons le lecteur.

A la suite de cette communication, le Congrès a émis le vou suivant, présenté par $M$. Schwoh :

"Que le Gouvernement fasse procéder sans retal'd. et plus particulièrement dans les bassins du Rhône, de la Loire, de la Garonne et de l'Adour, à une étude rapide permettant de fixer les principaux emplacements favorables à des réservoirs d'étiage, d'évaluer approximativement leur prix de revient, leur contenance, et les forces hydrauliques qu'ils rendraient disponibles.

( Cette enquête serait faite sur place par les services compétents, et les résultats en seraient publiés aussitôt,afin de permettre aux initiatives privées de puiser dans cette publication des indications précieuses".

Dans laprès-midi, M. Forgne, industriel à Toulouse, a donné lecture de son rapport sur les Institutions favorables au developpement de la batellerie.

M. Forgne étudie d'abord les Associations de bateliers. Il n'en existe que trois dans toute la région du Midi : l'une sur l'Isle, l'autre sul le Canal latéral, la troisième sur le Canal du Midi. Elles ne sont pas moins rares dans les autres parties du réseau navigable français. Il serait désirable quiune Union centrale pût se constituer en France à l'image de la vaste Association qui existe en Allemagne pour le développernent de la navigation fluviale allemande.

A ce sujet, M. Mallet rappelle qu'il existe déjà à Paris une Association, portant le titre de Chambre Syndicale de la Marine, qui paraît devoir répondre aux besoins signalés par l'orateur.

M. LAFFitTe signale qu'il a eu l'occasion d'étudier sur place le fonctionnement de l'Union centrale allemande. Cette Association a été créée par Bismarck. L'empereur s'y intéresse. Elle est bien le lien, si l'on veut, des Sociétés qui sefforcent de favoriser le développement de la navigation fluviale dans tout le pays allemand, mais elle comprend surtout un personnel d'industriels, de grands capitalistes, d'hommes de science.

M. Foigne étudie ensuite la question très complexe des écoles de batellerie. Il cite une société, l'En fance Bateliére, qui a pour but la création, dans les centres de navigation, d'interuats, d'écoles et de tous établissements destinés à l'éducation et à l'enseignement des enfants de mariniers. Au surplus, l'orateur'n'entend lemander au Congrès que de s'intéresser à l'institution de l'enseignement professionnel pour ces enfants.

M. Yves Guyot, ancien ministre des Travaux publics, et M. MALLET, font remarquer qu'il faudrait commencer par donner l'instruction primaire aux enfants des mariniers. Malheureusement, la question parait presque insoluble. Les enfants vivent auprès de leurs parents dans les péniches. Va-t-on les envoyer dans des internats pour leur apprendre à lire, à écrire, à compter? C'est impossible.

Il faudrait pourtant faire quelque chose. Un entrepreneur de transport du Centre, M. MaHaut, qui occupe un grand nombre de bateliers, signale que la plupart d'entre eux ne savent ni lire ni écrire, et sont incapables de signer leurs feuilles de service.

Enfin, M. Foigne étudie la question des Syndicals de garantie en matière de travaux d'amélioration ou de créa tion des voies navigables. Ces Syndicats paraissent d'une constitution difficile à l'orateur, s'il s'agit de recruter dans la région des souscripteurs qui se portent garants, vis-a. vis de l'Etat, des sommes à débourser pour créer ou améliorer des voies navigables. Mais il croit que l'an pourrait autoriser des péages pour gager les emprunks contractés par les Chambres de commerce en vue de l'amélioration de ros rivières et canaux.

M. Mallet dit qu'il est inutile de charger les Chambres de commerce d'améliorer. Car, pour améliorer, il faut des ressources, or, saul les Chambres des ports maritimes, qui sont autorisées à percevoir certaines taxes, et quelques rares Chambres, telles que celles de Paris et de Lyon, la plupart des autres n'ont pas un centime en caisse, quelles garanties peuvent elles donc hien offrir aux prêteur's?

A la suite de la communication de M. Foigne, le Congres émet les voeux suivants:

"Qu'une Association générale de tous les intéressés, professionnels ou non, soit créée en France, pour le développement de la Navigation Intérieure avec siège central à déterminer $\gg$;

«Que les Municipalités, Chambres de Commerco, Asso. ciations compétentes, et corps constitues, soient priés de donner leur concours pour étudier l'institution d'un enseignement professionnel de la Batellerie $»$;

"Que les Chambres de Commerce de la région examinen la possibilité d'une entente entre elles et l'Etat, en vue do l'amélioration des voies méridionales actuelles, et de la création de voles nouvelles de Navigation Intérieure $n$.

Le samedi matin, 20 juillet, M. MALLET, membre de h Chambre de Commerce de Paris, traite de la Concurren des Chemins de fer et des voies navigables.

10 La concurrence des voies d'eau et des voies ferrées est-elle profitable à l'industrie et au commerce? L'orateu expose une série de faits particuliers, qui lui permettenld conclure que, dans de nombreux cas, la navigation fluviale peut, ou pourra lutter à armes égales contre le chemin do fer. Presque toujours elle constitue une concurrence donl il doit tenir le plus grand compte; et toujours elle est un stimulant qui l'incite à progresser.

2o Est-il de l'intérêt général de développer la concurrence entre les voies d'eau et les voies ferrées? Sı le chemin d fer s'abstient, soil par prudence spontanée, soit par suite de l'opposition gouvernementale, l'action concurrente cesse.Sh au contraire, le chemin de fer veut faire ceuvre de concurrent, et que les pouvoirs publics ne l'entravent pas, n'osan pas faire obstacle à une mesure utile au commerce etá l'industrie, il peut en résulter des sacrifices très lourds pour lui, et peut-être menacants pour les finances publiques.

$3^{\circ}$ Quels sacrifices l'Etat doit-il faire pour favoriser th concurrence des deux voies? Si l'on veut édifier une ceuvit fluviale réellement profitable au pays, une ceuvre qui rend́ sincèrement plus économiques certains transports et qui puisse faire aux voies ferrées une concurrence loyale, il faut que les efforts portent sur un nombre restreint d'ops rations, scrupuleusement choisies, et douées de vitalité. Ne nous effrayons pas trop du rétablissement des péages. Sur une voie très fréquentée, ils pourront à la fois être de talts minime et supportés aisément. Il est préférable de payet des pêages modiques, et de jouir d'une voie très avantageuse, que de naviguer sans taxes sur une vole médiocr ou insulfisante. Au surplus, il devra être établi que tous los péages percus seront exclusivement affectês au service de la voie qui les aura produits.

$4^{\circ}$ Est-il possible de trouver un terrain d'entente entre tos deux modes de transport? L'Etat serait très bien inspiré el négociant avec les Compagnies de chemins de fer. En attendant, on peut progresser vers le but désiré. On a fall un pas de quelque importance le jour où a été votée la lon qui a décidé que, dorénavant, les détenteurs de nouvelles concessions de chemins de ler seraient tenus a raccordet 
d'autres établissements que les mines et les usines. On en ferait encore un intéressant en raccordant tous les chemins ce fer secondaires et en organisant solidement le camionnage par les voies de tramways. Cette mesure, souvent très utile, favoriserait particulièrement la navigation, en la reliant aux destinataires distants de quelques kilomètres.

M. Dubouchalld, président de la Chambre de commerce d'Angoulême, présente, au sujet du raccordement de la ligne de l'Elat avec le port de la Charente, une curieuse observation qui provoque l'hilarité générale. Ce raccorde. ment avait été promis par l'Etat, inscrit dans le contrat intervenu. Quand Angoulême en a réclamé la réalisation, l'Etat lui a répondu : « Le raccordement! Mais il est fait! Il est fait par le raccordement de la section de Rueil ». or, cette ligne spéciale se termine sur la Charente par une grue, destinée à charger les canons sur les gabares. Voilà ce que l'Etat appelle un raccordement. La grue en question charge des canons de 100 tonnes, mais Angoulême ne peut charger la plus petite barrique d'eau-de-vie sur sa rivière!"

Sur la proposition de MM. REY, BoIlÈve et ARMAND Jaçues, délégués du Syndicat National pour l'amélioration des transports, le Congrès émet le vou suivant :

« $1^{\circ}$ Que les pouvoirs publics cherchent le moyen pratique d'obliger les Compagnies de chemins de fer à ne mettre aucun obstacle au raccordement de leurs lignes avec les voies fluviales;

"20 Que le Gouvernement résolve définitivement et complètement la question des raccordements entre les voies ferrées et les voies navigables, soit en cherchant la solution par voie contentieuse, soit en négociant avec les Compagnies

Avant de se séparer, les Congressistes ont décidé que le deuxième Congrès National de Navigation Intérieure se tiendralt en 1909 à Nancy, à l'occasion de l'Exposition du Conmerce et de l'Industrie qui aura lieu à cette époque dans cette ville.

\section{H. BELLET.}

\section{NOTE AINITEXE}

Du 9 au 12 octobre, s'est également tenu à Bordeaux le Congrès National des Travauxx Publics. Ce Congrès a émis divers Vœux, se rapportant à la navigation intérieure, que nous croyons intéressant de reproduire ici.

\section{Sulure des ports, voies navigables et chemins de fer.}

Le Congrès : "Considérant que le trafic des marchandises entre l'Europe centrale et l'Amérique se déplace au profit des ports étrangers (Gênes, Anvers, Rotterdam, Hambourg), causant ainsi un préjudice à notre marine, à nos ports, nos chemins de fer, notre industrie et notre commerce; $)$

Emet le vœu : « 10 Que nos grands porls nationaux soient réunis à notre réseau de navigation intérieure, el mis en état de recevoir les grands navires transatlantiques et les baleaux de navigalion intérieure;

" $2^{\circ}$ Que les distances kilomélriques par voies ferrées qui séparent nos grands ports nationaux du 'cèntre de l'Europe soient réduites par la création de ports fluviaux intérjeurs associés, et que les marchandises en provenance ou à destination de la mer, transitant par les ports fluviaux, jouissent, à partir de ces ports, de tarifs spéciaux de transbordement; ces tarifs devraient toujours être sensiblement inférieurs à ce que donnerail l'application des barêmes de tarif intérieur des compagnies de chemins de fer ».

\section{Voie navigable de Nantes $\dot{a}$ Briare.}

Le Congrès: "Considèrant que le réseau navigable français présente dans la région de la Loire une lacune d'envicon 300 kilomètres entre Briare et Nantes ;

"Que cette lacune est préjudiciable, non seulement aux habitants, commercants et industriels de la région, mais à ceux de toules les parties de la France que l'établissemerit d'une voie d'eau entre Nantes et Briare mettrait en communicalion, par un moyen de transport économique, avec toute la vallée de la Loire et nos ports de l'océan ;
«Considérant que le projet de cette voie, soit par la création d'un canal latéral, soit par la mise en navigabilité de la Loire, est à l'élude depuis plus de trente ans ;

" Que l'état actuel de notre marine marchande et de nolre industrie des transports fluviaux rend éminemment désirable la prompte solution des questions intéressant nos ports de com merce maritimes et intérieurs ;

"Que, dans ces conditions, il convient, en se plaçant exclusivement au point de vue de l'intérêt général, de hâter la création d'une des voies navigables nécessaires à notre expansion commerciale industrielle ";

Emet le vœu : "Que les études en cours des diverses solutions pour la création de cette voie navigable soient poussées le plus activement possible, et qu'il soit fait appel à l'iniliative privée pour sa réalisation ».

\section{Canal latéral au Rhóne.}

Le Congrès: «Considérant que les travaux exécutés sur le Rhone, si importanls qu'ils soient, ne suffisent pas a assurer une navigation régulière et économique, et que, par suite, la création d'un canal latéral de navigation entre Arles et Lyon s'impose ;

"Que, d'autre part, les populations du Midi réclament de nouveau, avec une insistance bien légitime, la création de canaux d'irrigation propres à assurer la mise en valeur de territoires importants en y permettant la polyculture ;

«Qu'il y a lieu, encore, de prévoir la possibilité d'utilisation des eaux du Rhòne pour la créatıon de forces molrices imporlantes

“ Mais considérant qu'il n'y a pas nécessairement connexité entre les trois entreprises, et que des syndicats privés d'initiative se sont formés séparément pour l'élude du canal de navigation et pour celle des canaux d'irrigation »;

Emet le vœu : : 10 Que les études relalives aux canaux de navigation, d'irrigation et d'utilisalion des forces motrice du Rhône solent conduites le plus rapidement possible ;

«20 Qu'il soit fait appel à l'industrie privée pour la réalisation de ces projets".

\section{Jonction de la Garonne, de la Loire et du Haut-Rhône.}

Le Congrès: "Considérant l'utilité que présenterait la création d'une voié navigable de jonction entre la Garonne et la Loire ;

"Que, d'autre part, il y aurait grand intérêt à relier la navigalion du Rhone au réseau navigable suisse $\epsilon$ t au Rhin »;

Emet le vœu: :Que les études relatives à ces deux importantes questions soient puursuivies, et fassent l'objet de mémoires à sou mettre au prochain Congrès des Travaux Publics ».

\section{Reboisement et régularisation des rives des cours d'eau.}

Le Congrès : "Considérant qu'il est urgent de chercher à mettre un terme aux ravages des cours d'eau à régime torrentiel et à fond mobile, et alténuer les variations de leur débit ";

Emet le vœu : a 10 Que les engazonnements et les reboisements des hautes vallées soient poussés activement;

" $2^{\circ} \mathrm{Que}$ les pouvoirs publics provoquent et encouragent, par des subventions, l'exécution de travaux propres à fixer les rives et, la pente de ces cours d'eau $»$.

\section{Personnalilé civile des grands travaux publics.}

Le Congrès: «Considérant que la centralisation à Paris de toutes les initiatives, et de toutes les décisions, en matière de grands travaux publics et, notamment, en ce qui concerne les ports de commerce et leurs voies d'accès navigables, est la cause direcle de l'arrêt presque complet desdits travaux et, par suite, de l'infériorité manifeste de nos ports et de la décadence de nutre marine marchande :

"Que les intéressés directs de la région sont bien placés pour se rendre comple de l'utilité et de l'urgence de ces travaux, et que l'initiatrve doit aller de pair avec les charges et les responsabilités

"Que l'étude des projets subit, par suite de la centralisalion hiérarchique des services officiels, des lenteurs interminables allant de dix.huit mois au minimum à quatorze ans pour le Havre ;

"Cue le cout d'exécution des travaux dépasse considérablement celui des entreprises privées ;

iq Que les sommes prélevées sur le budget général en faveur des travaux publics sont, étant données les obligations essentielles et multiples de l'Etat, loin de correspondre aux exigences de notre outillinge national; 
"Que cette situation budgétaire ne paraît pas, quelle que soit la bonne volonté des pouvours publics, devorr se modifier de longlemps;

"Que ces crédits, déjà insuffisants, risquent, en outre, d'être répartis d'une façon non conforme aux intérêts généraux du pays;

"Que les sacrifices consentis par l'Etat se traduisent par l'allocation de crédits échelonnés sur un des exercices successifs, ce qui constitue souvent une nouvelle cause de retard et de cherté dans l'exécution des travaux »;

Emet le vœu : "Que l'initiative, l'étude, l'exécution et l'administration des travaux des ports maritimes el fluviaux, canaux ou autres grands travaux publics puissent ètre confiées à des collectuvités locales ou régionales, dolées de la personnalité avile. notamment à des syndicals aulonomes, dans lesquels seroni représentés les grands intérêts locaux, régionaux ou nationaux «.

\section{Mourements des Liguides dans les copps creux de pérolution Par le Professeur Dr Prasil (*)}

En traitant les problèmes d'hydrodynamique par les équations fondamentales de Lagrange, ou d'Euler, on rencontre des difficultés, provenant de ce que l'intégration des équations aux différentielles partielles, auxquelles on est conduit, n'est souvent pas possible par les méthodes con nues jusquà présent.

Les recherches faites pour des liquides incompressibles se rapportent, d'une part, aux mouvements dans lesquels les vitesses sont déduites de l'existence d'un potentiel des vitesses, et, d'autre part, de la théorie des mouvements tourbillonnaires. Elles sont généralement faites en se servant d'un système de coordonnées rectilignes et orthogonales; Grashof, dans ses leçons théoriques sur les machines (Theoretischen Maschinenlehve, ${ }^{\text {er }}$ vol.pages $3 g 8$ et suiv.) a préconisé, pour les recherches générales des mouvements des fluides suivant des trajectoires données, l'emploi de coordonnées curvilignes et orthogonales; quelques cas spéciaux seulement ont été traités par les coordonnées cylindriques. La recherche des écoulements dans un plan a conduit à la solution du problème dit « à deux dimensions ", et, par suite, à la déterinination de la forme des filets liquides. Si tant est que ces recherches soient propres à déterminer une série de mouvements des liquides, elles sont, néanmoins, inapplicables dans la plupart des cas qui se présentent actuellement; elles ne donnent généralement que des bases de comparaison.

Parmi les problèmes d'hydrodynamique qui ont une importance particulièrement immédiate dans la construction des machines, on trouve, entr'autres, ceux qui se rapportent aux mouvements des fluides dans des canaux. En général, on a à faire, ou bien à des canaux constitués par des corps creux de révolution (tuyaux, buses), ou bien à des canaux groupés de façon à former des corps analogues (distributeurs et roues de turbines et pompes centrifuges).

Il semble donc que l'on pourrait, dans les deux cas, simplifier les déductions analytiques pour la recherche du mouvement des fluides, en transformant en coordonnées cylindriques (admettant comme axe principal l'axe de révo-

(*) Traduction des articles parus dans la Schweizerische Bauzeitung, vol XLI; no 19,2I, 22, 25 et 26, par M. Georges Servière, ancien élève de l'Ecole Polytechnique, ingénieur aux Ateliers de constructions Neyret-Brenier et Cie de Grenoble. tution) les équations fondamentales citées au début, e! établies en coordonnées cartésiennes.

La présente étude fait connaître les résultats de ces recherches; on les a appliqués en premier lieu à la déter. mination des méridiennes des tuyaux d'aspiration des lurbines.

Ce sujet se rattache à la discussion des équations fonda. mentales du mouvement des fluides parfaits incompres. sibles, exposée par Grashof dans ses Theoretischen Maschinenlehre (I vol. Page 386 et suiv.).

\section{I. - TRANSFORMATION DES ÉQUATIONS FONDAMENTALES}

La transformation en question pourrait se faire analytiquement, en prenant l'axe des $z$ pour axe principal, et en

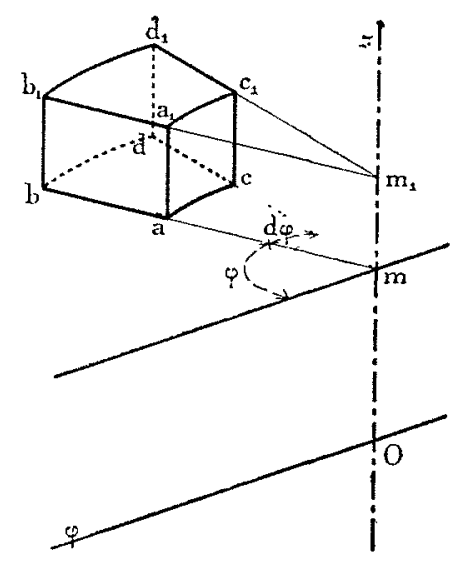

Fig I. remplaçant les coordonnées d et $\gamma$ par les coordonnées polai. res $r$ et $\varphi$. II nous semble cependant plus simple d'établi directement ces équations.

Considérons (fig. I), dan un anneau, un élément $d$ : volume abcda $b_{1} c_{1} d_{1}$ dont: sommet a, a pour coordonnt cylindriques $\%$ et $\overrightarrow{\mathrm{om}}=$; $\overline{\mathrm{ma}}=r$.

Les longueurs des côtés dit l'élément sont :

$$
\begin{aligned}
& \overline{\mathrm{ac}}=\overline{\mathrm{a}_{1} \mathrm{c}_{1}}=r \mathrm{~d} \varphi \\
& \overline{\mathrm{bd}}=\overline{\mathrm{b}_{1} \mathrm{~d}_{1}}=(r+\mathrm{d} r) \mathrm{d} \varphi \\
& \overline{\mathrm{ab}}=\overline{\mathrm{a}_{1} \mathrm{~b}_{1}}=\overline{\mathrm{cd}}=\overline{\mathrm{c}_{1} \mathrm{~d}_{1}}=\overline{\mathrm{d}} r \\
& \overline{\mathrm{aa}}=\overline{\mathrm{bb}_{1}}=\overline{\mathrm{cc}_{1}}=\mathrm{dd}_{1}=\overline{\mathrm{mm}_{1}}=\mathrm{d} z
\end{aligned}
$$

La projection de l'élément de volume sur un plan perpendiculaire à l'axe des $z$ donne la fig. 3 ; la projection

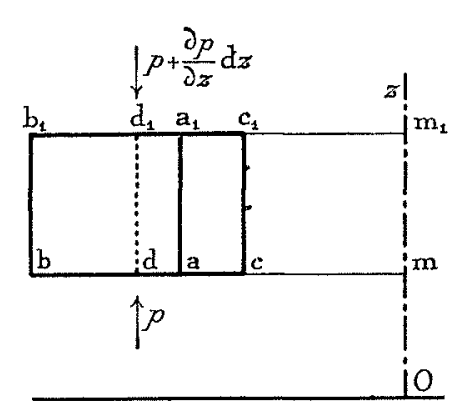
verticale donne la fig. 2 .

La masse du fluide qui rem plit l'élément de volume est

$$
\mathrm{d} M=\frac{\gamma}{g} r \mathrm{~d} \varphi \mathrm{d} r \mathrm{~d} z
$$

oú $\gamma$ est le poids de l'unitéd volume du fluide, et $g=g^{m f}$ O par seconde est l'accélérati de la pesanteur.

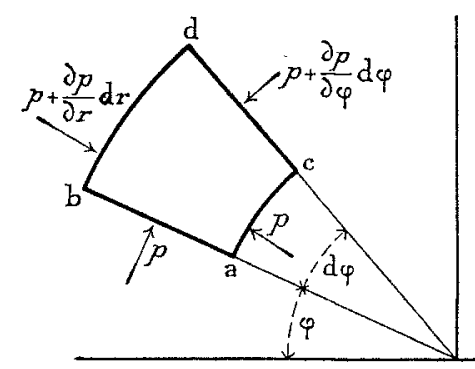

lig. 2 ct 3 .
Nous représenterons les for ces dues à la masse, pour l'u nité de masse, par :

$R$, dans la direction de $\mathrm{r}$ suivant le rayon, et positire ment vers l'extérieur;

$Z$ dans la dircction de l'as des $z$, positivement dans lesen des fig. 1 et 2 ;

$U$ perpendiculairement à et $Z$, et positivement dans lesens de la rotation des aiguill d'une montre. 